\title{
A direct approach to control short term population dynamics in time series studies
}

\section{Stefano Zauli Sajani, Fabiana Scotto, Paolo Lauriola}

J Epidemiol Community Health 2005;59:985-986. doi: 10.1136/jech.2005.033654

Background: Short term population dynamics is an important issue in several epidemiological studies. Usually, calendar time or dummy variables are used to control indirectly for this confounding. This study tested a direct method.

Methods: The study compared as proxy variables of population dynamics the summer 2003 data of cooking gas consumptions, solid urban waste production, and television access for the municipality of Bologna (Italy).

Results: Solid urban waste production and television access data showed similar trends. Considerably different were the $>65$ year olds estimates with respect to total population based on television access.

Conclusions: Television access data are probably the best indicator in the estimates of population dynamics in large or densely populated areas, especially because of the possibility of stratifications with respect to age.

S hort term population dynamics is an important issue in several epidemiological studies. Health risks, estimated using the resident set as exposed reference population, could be heavily affected by population displacements, which show a pronounced trend especially in middle latitude regions during summer. Heat and ozone are important environmental examples of such risk factors. ${ }^{1-5}$ Time series studies usually take into account only mortality and illness of residents who die or become ill in the study area. This choice aims to reduce errors in the estimate of the exposure dose to the risk factors under study but introduces errors in the definition of the number of exposed persons. The approach used in literature to face this problem is to insert in models (for example, generalised additive models) calendar time variables or dummy variables such as day of the week, season, holidays, or other such time indicators. Smoothers like loess smoother or natural splines are then often used to control the time series with respect to these variables. This methodology however is not satisfactory. First of all, it addresses two different factors that can influence the time variation of outcome occurrence and that should be studied separately: population displacements and services organisation (health and social care, shops opening). Furthermore, the variables are not independent and could probably not be considered one by one. Lastly, because of the large stochastic component in the outcome variability, it is unlikely that a model can capture the real population fluctuations with an indirect approach based on the effect of a set of time indicators on the health variables. In this work, we tested a direct approach to address population displacements using as indicators cooking gas consumption, solid urban waste production, and television access data.

\section{METHODS}

We compared the summer 2003 data of cooking gas consumptions, solid urban waste production, and television access referred to the municipality of Bologna, a city of 371000 inhabitants in the north east of Italy. Daily data of cooking gas consumption and solid urban waste production were given by Hera, the main service factory of the area. Even if waste composition changes during the year, it is nearly uniform during summer season. Daily television access was defined as the number of persons who switched television on at least once during a day. Data were derived from AUDITEL (AGB Italia), the Italian telvision access monitoring system. The system is based on the remote control of a panel of persons of different age, sex, social, and economic status: this permits stratified estimates, first of all by age. The 2003 water and electricity household consumption in the study area were unavailable.

\section{RESULTS}

Figure 1 shows the comparison between the summer 2003 trends of seven day moving averages of cooking gas consumption, solid urban waste productions, and television access. Each variable was "normalised" dividing daily values by the average of the first week's data. The best agreements were found between solid urban waste production and television access. The minimum was on 15 August. In this period solid urban waste productions and television contacts were between $50 \%$ and $60 \%$ of the usual value. A similar trend appeared in cooking gas consumption, with an even more pronounced minimum (nearly $40 \%$ of the usual value). Figure 2 compares the estimates of the total population and the $>65$ year olds based on television data. The $>65$ population in August was about $70 \%-80 \%$ of the $>65$ residents; this percentage was $40 \%-45 \%$ for $<65$ years old.

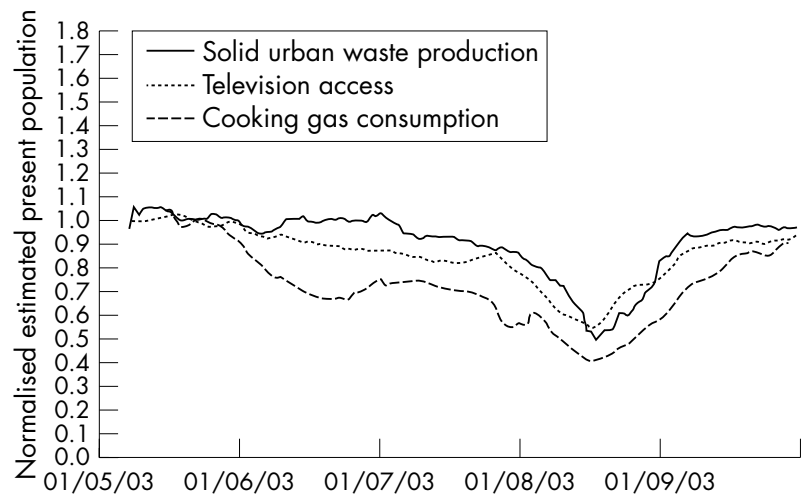

Figure 1 Seven day moving averages of solid urban waste production, cooking gas consumption, and television access during summer 2003. The data are "normalised" dividing daily values by the average of the first week's data. 


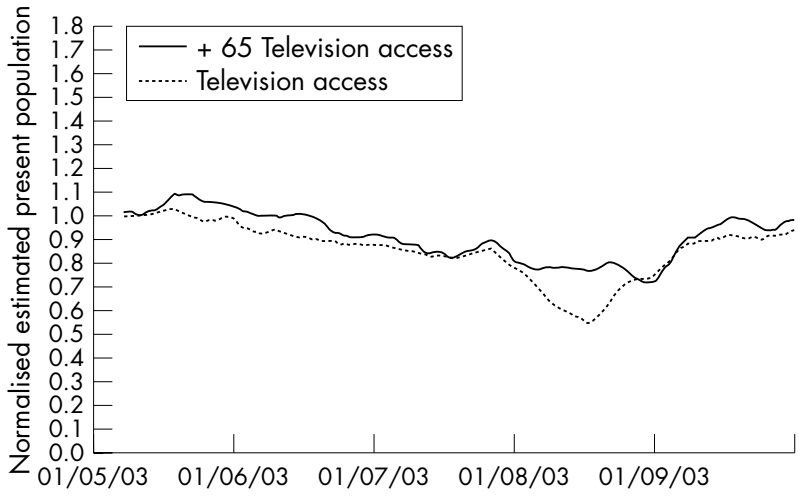

Figure 2 Seven day moving averages of total and +65 years old television accesses during summer 2003. The data are "normalised" dividing daily values by the average of the first week's data.

\section{What is already known on this subject}

- Short term population dynamics is an important issue in several epidemiological studies

- Calendar time or dummy variables are used to control indirectly for this confounding.

\section{What this study adds}

We tested a direct method based on the analysis of water and cooking gas consumptions, solid urban waste production, and television access. Television access data seem the most appropriate indicator of the present population.

The analysis of regional data that take into account both rural and urban data showed even more noticeable differences between population estimates in the different age stratifications.

\section{DISCUSSION}

Even though we had no reference value of the number of people really present, we can argue that the consistency in the trends between solid urban waste production and television access enables these variables to be considered as good indicators of the total population present during the summer season. Cooking gas consumption could also be a useful indicator but these data are affected by the error connected with the estimate of the cooking part of the total gas consumption. The cooking part was calculated from the total gas consumption by applying a monthly coefficient given by the service factory. Household electricity and water consumptions, not available for this study, are thought to be related to meteorological variables (especially temperature) and so probably unsuitable for population variation estimates. Even if waste and television access data seem the best population proxy variables, problems arise in using these

\section{Policy implications}

Present population estimates permit better quantification of the expected health outcomes and a proper organisation of health services and watch/warning systems.

data. Solid urban waste collection, for example, is not easy to differentiate with respect to administrative contours. Television data are usually not public data; nor are they statistically significant for small towns or sparsely populated areas. There are also difficulties in obtaining estimates of the present population up to the daily scale, especially for weekend days. Urban waste data are strongly biased because collection is scanty (this problem also affects Monday data) and television data may also be misleading because the criterion of one television access during the day is probably not satisfactory. A person may switch on the television on Saturday morning and then go away and come back on Sunday evening, when he may switch it on again. In this case the criterion of presence adopted in this study would classify them as present for both Saturday and Sunday. This problem might be partially solved by adopting specific criteria for weekend days based on the analysis of hourly data. In conclusion, television access data could probably be considered the best indicator of summer population dynamics, not only because of the possibility of stratifications with respect to age and, perhaps, socioeconomic status but also because these television control systems are present in most places in the developed world. However, the use of data on human activities as indicators of short term population dynamics should be investigated in different countries and cities before application in epidemiological studies and public policies.

\section{Authors' affiliations \\ S Zauli Sajani, F Scotto, P Lauriola, Environmental Epidemiology Unit, ARPA Emilia Romagna, Modena, Italy \\ Funding: none. \\ Conflicts of interest: none.}

Correspondence to: Dr S Zauli Sajani, Environmental Epidemiology Unit, ARPA Emilia Romagna, Viale Fontanelli 23, 41100 Modena, Italy; szauli@epam.arpa.emr.it

Accepted for publication 30 April 2005

\section{REFERENCES}

1 Biggeri A, Bellini P, Terracini B. Meta-analysis of the Italian studies on shortterm effects of air pollution-MISA 1996-2002. Epidemiol Prev 2004;28:1-100.

2 Bell ML, McDermott A, Zeger SL, et al. Ozone and short-term mortality in 95 US urban communities, 1987-2000. JAMA 2004;292:2372-8.

3 Curriero FC, Heiner KS, Samet JM, et al. Temperature and mortality in 11 cities of the eastern United States. Am J Epidemiol 2002;155:80-7.

4 Goodman PG, Dockery DW, Clancy L. Cause-specific and extended effects of particulate pollution and temperature exposure. Environ Health Perspect 2004; 112:179-85.

5 Gryparis A, Forsberg B, Katsouyanni K, et al. Acute effects of ozone on mortality from the "Air pollution and health: a european approach" project. Am J Respir Crit Care Med 2004;170:1080-7. 